\section{Categorizing Hydrogen Bonding and Other Intermolecular Interactions}

The aim of this project is to take a comprehensive look at intermolecular interactions and classify them and to give a modern definition of the hydrogen bond, taking into account all current experimental and theoretical information, and including hydrogen bonded systems both in gaseous and condensed phases as well as in chemical and biological systems. This report summarizes the activities of the task group for this project over the last two years.

A workshop was held in Pisa, Italy, from 5-9 September 2005, in which 11 task group members and 11 other experts each gave 30-minute presentations on their recent work relevant to the project. The details of this workshop are available on the project webpage.

On 9 September 2005, the task group met to discuss the proceedings of the Pisa workshop and to produce an interim report. There was a unanimous view among the task group and workshop participants that there is no single physical force that can be characterized as hydrogen bonding or van der Waals interaction. This may be contrasted with covalent bonding (as in the $\mathrm{H}_{2}$ molecule), ionic bonding (as in $\mathrm{KCl}$ molecule in the gas phase), and London dispersion forces (as in $A r_{2}$ ). Hydrogen bonding is used by various scientists to describe interactions in extremes that can be summarized beautifully by an isoelectronic series, $(\mathrm{FHF})^{-}, \mathrm{HF} \cdot \bullet \mathrm{HF}$, and Ne・•HF, suggested by Legon. In (FHF) ${ }^{-}$, we have a very strong hydrogen bond with a binding energy ${ }^{1}$ of $167 \mathrm{~kJ} \mathrm{~mol}^{-1}$, that borders a covalent bond. In HF••HF, we have a typical hydrogen bond with a binding energy ${ }^{2}$ of $19 \mathrm{~kJ} \mathrm{~mol}^{-1}$, that is dominated by electrostatic forces. Finally in $\mathrm{Ne} \cdot \bullet \mathrm{HF}$, we have a weak interaction with a binding energy 3 of $1 \mathrm{~kJ} \mathrm{~mol}{ }^{-1}$, dominated by dispersive and inductive forces. The task group came up with an interim recommendation for the definition of hydrogen bonding and classification of intermolecular forces. This report was discussed extensively within the task group and with participants through e-mail.

A core group of Elangannan Arunan, Gautam Desiraju, Roger Klein, and Joanna Sadlej met in Bangalore from 18-22 September 2006 to finalize the recommendation. Steve Scheiner, co-chairman of the task group, participated by video-conferencing. On 18 September, a one-day meeting was organized at the Indian Institute of Science in Bangalore, with talks by the core group and five other experts. Meeting details are available at <http://ipc.iisc.ernet. in/ arunan/Bangalore_iupac_meet2.html>.

Arunan gave a brief introduction to the project and pointed out the diverse views existing in the literature on the definition of hydrogen bonding and on van der Waals interactions. Desiraju gave the first talk, titled "The $\mathrm{C}-\mathrm{H} \cdot \cdots \mathrm{O}$ and Other Weak Hydrogen Bonds: From Crystal Engineering to Virtual Screening." His talk summarized the voluminous literature existing in the domain of "weak" hydrogen bonds. In particular, he pointed out that $\mathrm{C}-\mathrm{H} \bullet . \cdot \mathrm{F}$ interactions can be unambiguously identified from the Cambridge Crystal Structure Database, if the search is limited to fluorobenzenes rather than all molecules containing $\mathrm{C}-\mathrm{H}$ and $F$, and other atoms. Guru Row presented results on experimental electron densities of substituted coumarins. Using Koch and Popelier's criteria ${ }^{4}$ he showed that the $\mathrm{C}-\mathrm{H} \cdot \cdots \mathrm{O}$ interactions found in these systems could be classified as hydrogen bonding, but the $\mathrm{C}-\mathrm{H} \bullet \bullet$ p interactions would be van der Waals. The key criterion that leads to this difference is the mutual penetration of atoms that depends crucially on the assumed van der Waals radii of the two bonding partners. He also presented results on organic fluorine compounds that show a bond critical point between $\mathrm{C}-\mathrm{F}$ groups implying $\mathrm{C}-\mathrm{F} \cdot \bullet \cdot \mathrm{F}-\mathrm{C}$ contacts.

Sadlej presented theoretical results on IR and NMR spectral properties of water clusters as a doorway to the mysteries of liquid water. She presented the $\mathrm{OH}$ stretching frequencies for $\left(\mathrm{H}_{2} \mathrm{O}\right)_{n}(\mathrm{n}=3=12)$ and compared them with available experimental results. She presented 170 and $1 \mathrm{H}$ chemical shifts and $1 \mathrm{~h} \mathrm{~J}_{\mathrm{OH}}$ and $2 \mathrm{~h} \mathrm{~J}_{\mathrm{OO}}$ spin-spin coupling constants for several clusters. She ended her talk with a question: Do these clusters really exist in liquid water? Sathyamurthy gave a talk with a title "Hydrogen bonding without borders." He showed that the binding energies for dimers have a linear relationship with the electron densities and 
the Laplacian at the bond critical points for "hydrogen bonded" complexes. He discussed the p-p interactions in a series of aromatic compounds with and without a permanent dipole moment. Jemmis gave a lecture on "The Long and Short of Weak Hydrogen Bonds." He presented electronic structure calculations on several $\mathrm{C}-\mathrm{H} \cdot \cdots \mathrm{Y}$ hydrogen bonded systems. Optimization of $\mathrm{C}-\mathrm{H}$ distance and the energy as a function of $\mathrm{H} \cdot \cdots \mathrm{Y}$ distance revealed that the minimum in the binding energy need not be at the minimum for the $\mathrm{C}-\mathrm{H}$ distance. Hence, the $\mathrm{C}-\mathrm{H}$ bond length at the energy minimum could be longer, shorter, or unchanged compared to the $\mathrm{C}-\mathrm{H}$ distance in the unperturbed monomer. This could explain both red and blue shifting of $\mathrm{C}-\mathrm{H}$ groups that are observed in hydrogen bonded systems.

Klein gave a talk on "Characterizing Hydrogen Bonding: Creation and Genesis." He argued that a bond-critical point must be present between the $\mathrm{H}$ and the acceptor atom. He pointed out the inadequacy of single van der Waals radii of atoms in confirming/ ruling out hydrogen bonding. He also showed that the attractive hyper-conjugative effects exceed Pauli exchange (steric) repulsion at the optimum geometry for hydrogen bonded systems. Naresh Patwari presented a talk titled "Is Dihydrogen Bonding Analogous to Hydrogen Bonding?" He showed the strong correlation between proton affinity of the acceptor and the frequency shift observed in $\mathrm{O}-\mathrm{H}$ stretching frequency in a series of hydrogen bonded complexes. However, for dihydrogen bonded complexes, this correlation worked only with an empirical correction of 0.84 (i.e., dihydrogen bonded systems are similar to hydrogen bonds, but 16 percent weaker).

In K.S. Viswanathan's talk on "Hydrogen Bonds in Cryogenic Matrices," he presented infrared spectra of several hydrogen bonded complexes observed in a matrix and showed that the experimental frequency shifts had a good correlation with the computed frequency shifts and also the binding energies of the hydrogen bonded complexes. Arunan gave the last talk of the day, titled "Hydrogen Bond Radii: From Microwave Spectroscopic, ab initio and AIM Studies." He presented microwave spectroscopic results on several $\mathrm{H}_{2} \mathrm{O}$ and $\mathrm{H}_{2} \mathrm{~S}$ complexes and showed the structural similarities between these complexes. He also showed that the $\mathrm{H} \cdots \bullet Y$ distance could be written as a sum of hydrogen bond radius for $\mathrm{X}-\mathrm{H}$ donor and an acceptor radius for $Y$. He presented theoretical results from $\mathrm{ab}$ initio and AIM calculations and showed that both the donor and acceptor radii increase from strong to medium to weak hydrogen bonds. He presented a set of "hydrogen bond radii" that may be used instead of the single set of van der Waals radii for all atoms in confirming/ruling out the presence of hydrogen bonds.

There was a panel discussion at the end that involved all the participants (about 100). The discussion centered on the use of experimental and theoretical electron density topology in confirming/ruling out hydrogen bonds. Arunan presented the evolving definition of hydrogen bonding and sought comments from everyone. After the meeting, the core group finalized the recommendation and started working on a manuscript that will justify its recommendation. This recommendation was circulated within the task group and was revised based on suggestions/criticisms. For hydrogen bonding, the proposed definition follows closely on the one given by Pimentel and McClellan. ${ }^{5}$ It was decided to propose a short definition and a list of criteria and characteristics for hydrogen bonding.

\section{References}

1. N. Elgobashi and L. González, J. Chem. Phys. 124, 174308 (2006).

2. W. Klopper, M. Quack, and M.A. Suhm, J. Chem. Phys. 108, 10096 (1998).

3. M. Meuwly and J.M. Hutson, J. Chem. Phys. 110, 8338 (1999).

4. U. Koch and P.L.A. Popelier, J. Phys. Chem. 99, 9747 (1995).

5. G.C. Pimentel and A.L. McClellan, The Hydrogen Bond, W. H. Freeman and Co., San Fransisco (1960).

For more information, or comments, contact Task Group Co-Chairmen Elangannan Arunan <arunan@ipc.iisc.ernet.in> or Steve Scheiner <scheiner@cc.usu.edu>.

\section{iin, www.iupac.org/projects/2004/2004-026-2-100.html.}

\section{Recommendations for Isotope Data in Geosciences}

A joint project between the Inorganic Chemistry and Analytical Chemistry Divisions of IUPAC and the International Union of Geological Sciences (IUGS) has been approved to evaluate radioisotope decay data used in the geosciences and planetary sciences for dating. Significant disparities exist between data used in these communities and those employed in physics and chemistry. The project will draw on complementary expertise represented in IUGS and IUPAC. Previous work by IUGS (Working Group "Decay Constants in Geochronology") and IUPAC (Commissions II.1 "Isotopic Abundance" and V.7 "Radiochemistry and Nuclear Techniques") will be included, harmonized, 